\title{
DISTRIBUTED AND THERMO-ACOUSTICALLY COUPLED MODELING FOR ACCURATE PREDICTION OF THERMAL NONLINEARITY IN PIEZOELECTRIC MEMS RESONATORS
}

\author{
Rahul Jhaveri, Ruochen Lu**, Songbin Gong ${ }^{* *}$, and Mattan Kamon ${ }^{*}$ \\ *Coventor, Inc., USA \\ **University of Illinois at Urbana-Champaign, USA
}

\begin{abstract}
In this paper, we employ for the first time a distributed and thermo-acoustically coupled modeling technique for thermal nonlinearity. The technique captures the true distribution of temperature throughout the resonator and its surrounding support structure. The resultant non-uniform temperature distribution modifies the stiffness coefficients in an acoustic simulation to directly compute the sensitivity of the frequency to power input. The technique results in new insights with respect to geometry, and enables geometric design tradeoffs to mitigate the effects of thermal nonlinearity. Our technique is compared to the lumped method in prior work and verified by measurements on two aluminum nitride resonators.
\end{abstract}

\section{INTRODUCTION}

Thermal nonlinearity has been identified as one of the key limiters on power handling and the primary mechanism for producing intermodulation in piezoelectric MEMS resonators [1][2][3]. Previous techniques to model thermal nonlinearity are either analytic [4] or lumped [1], neither of which can account for geometric details, and thus are limited in capturing detailed thermoacoustic coupling. For instance, the lumped model from [1], as shown Fig. 1, assumes that the resonator body is at a single temperature and the only significant thermal resistance to heat flow is through the anchors. This assumption breaks down as the length of the resonator grows such that there is a significant thermal resistance from the center of the resonator to the anchors. Similarly, if the anchors are widened to be on the order of the resonator width, the resonator body's thermal resistance is also on the same order as the anchors and cannot be neglected. While the thermal resistors could be adjusted or a different thermal circuit topology could be used, both require heuristic knowledge, and are approximations at best. Thus, it is challenging for heuristic models to capture the true thermal paths and be predictive for device performance optimization.

To improve, we employ a distributed and thermo-acoustically coupled modeling technique via the finite-element analysis (FEA). The coupled analyses determine the power coefficient of frequency (PCF) which directly relates the change in resonance frequency to the absorbed power. It is a more precise method because the nonuniformed heat generation and temperature profile across the resonator body is explicitly determined from the acoustic modeshape. The derived temperature profile is subsequently used to modify the stiffness for each meshed element and compute the resonance change via frequency domain FEA. Similar to the technique in [1], this work also captures the thermal nonlinearity by realizing that the shift in resonance frequency due to self-heating not only changes the amplitude of oscillation and thus the heat generated at a given frequency, but also alters the impedance mismatch seen by the source, further changing the heat generated. By coupling the feedback effect in thermal nonlinearity [1] with the PCF-based modeling method, the impact of thermal nonlinearity on the resonator admittance response is determined. Two aluminum nitride (AlN) resonators similar to those in [5] are measured, and results are compared with our modeled results to demonstrate the effectiveness

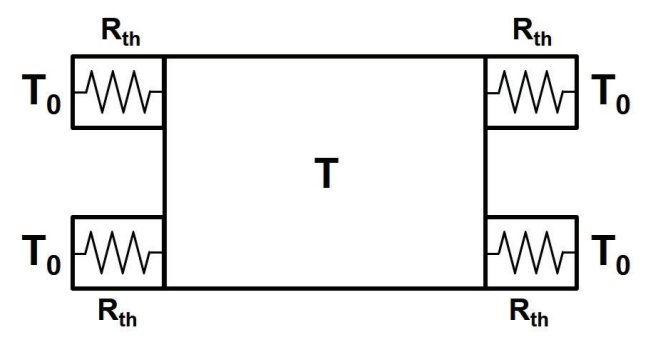

Figure 1: Lumped thermal model applied in [1]. The resonator body is at a single temperature, $T$, thermally coupled to ambient, $T_{0}$, through thermal resistors for the anchors.

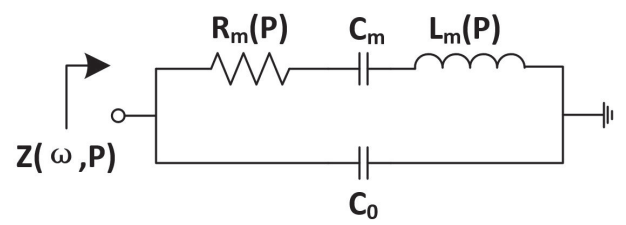

Figure 2: Equivalent Butterworth Van-Dyke model for the resonator showing power dependent circuit components.

of the developed model.

\section{MODELING METHODOLOGY}

In this section a top-down description of the modeling methodology is presented, starting with the origin of the nonlinearity.

\section{Absorbed Power}

The self-heating that shifts the resonance frequency and thus the impedance of the resonator, is a function of the steady-state absorbed power, $P$. That is, $Z=Z(\omega, P)$ where $\omega$ is the radian drive frequency. The absorbed power also obeys the standard relation for absorbed power of the load at an impedance mismatch:

$$
\frac{P}{P_{a v s}}=1-\left|\frac{Z(\omega, P)-Z_{0}}{Z(\omega, P)+Z_{0}}\right|^{2}
$$

where $P_{a v s}$ is the power available from a source with an impedance of $Z_{0}$. If the functional relationship $Z(\omega, P)$ can be determined, then Eq. 1 can be solved numerically for each frequency, $\omega$, to determine $P=P(\omega)$. The desired admittance from thermal nonlinearity of selfheating is $\mathrm{Y}(\omega)=Z(\omega, P(\omega))^{-1}$.In the next section we describe the determination of the function $Z(\omega, P)$.

\section{Resonator Input Impedance}

The impedance of a piezoelectric resonator near resonance is well modeled by the Butterworth Van-Dyke (BVD) circuit model shown in Figure 2. The components of the BVD circuit without selfheating $(P=0)$ can be determined from simulation or measurement of the resonance frequency $\left(\omega_{0}\right)$, the electromechanical coupling coefficient, $k_{t}{ }^{2}$, the static capacitance, $C_{0}$, and the quality factor, $Q$. To determine the circuit components under self-heating, note that 


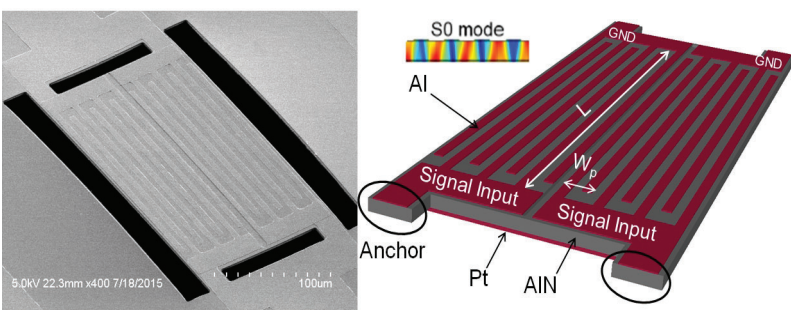

Figure 3: AlN laterally vibrating resonator used in this study and similar to [5].

for a given $P$, the self-heating in steady-state shifts the resonance frequency, thus $\omega_{0}=\omega_{0}(P)$, which implies the motional resistance, $R_{m}$, and motional inductance, $L_{m}$, are functions of absorbed power:

$$
\begin{aligned}
R_{m}(P) & =\frac{\pi^{2}}{8} \frac{1}{\omega_{0}(P) C_{0} k_{t}^{2} Q} \\
L_{m}(P) & =\frac{\pi^{2}}{8} \frac{1}{\omega_{0}(P) C_{0} k_{t}^{2}}
\end{aligned}
$$

Note that we assume $k_{t}^{2}$ and $C_{0}$ to be independent of self-heating as the temperature coefficients of piezoelectric coupling and permittivity of AlN are insignificant. $Q$, despite being observed to a weak function of power prior to thermal bifurcation [2], is also treated as a constant versus power for simplifying the discussion in this work.

Since the heat equation is linear, and assuming the temperature variation is small enough that the elastic stiffness varies linearly, the resonance frequency is linear with power and can be written in terms of a power coefficient of frequency (PCF):

$$
\omega_{0}(P)=\omega_{0}^{0}(1+P C F \cdot P)
$$

where $\omega_{0}^{0}$ is the resonance frequency at ambient temperature and zero input power $(P=0)$. In the next section, we describe the determination of PCF with distributed thermo-acoustic modeling via the finite-element method.

\section{Power Coefficient of Frequency}

In order to complete the determination of $Z(\omega, P)$, the power coefficient of frequency (PCF) as defined in Eq. 3 must be determined. To do so, we use the finite element method to compute $\omega_{0}^{0}$ and $\omega_{0}\left(P^{\prime}\right)$ for a given $P^{\prime}$ and use Eq. 3 to compute PCF. For simplicity of explanation, we assume the power lost to acoustic radiation to the substrate is negligible (we will address this in more detail below). Under this assumption, the absorbed power, $P$, generates heat proportional to the square of the magnitude of the local displacement. This is consistent with effects such as interfacial loss and thermoelastic damping. The local displacement will be proportional to the local eigenmode displacement and can be scaled appropriately to produce the correct net generated heat, $P$ ', for the local heat generation used in the thermo-acoustic modeling. The six steps of the method are as follows:

1. Compute zero-power (ambient temperature) resonant mode frequency $\omega_{0}^{0}$, and mode shape displacements, $\boldsymbol{u}(\boldsymbol{r})$, where $\boldsymbol{r}$ is a point inside the resonator

2. Apply volume heat flux, $q(\boldsymbol{r})$, to the thermal model $q(\boldsymbol{r})=$ $k|\boldsymbol{u}(\boldsymbol{r})|^{2}$ with $k$ such that the total heat applied is the given $P^{\prime}$

3. Solve the heat equation in the resonator, support structure, and substrate to determine temperature field, $T(\boldsymbol{r})$

4. Modify the local stiffness tensor: $c=c(T(\boldsymbol{r}))$

5. Recompute mode frequency using the distributed stiffness $c(T(\boldsymbol{r}))$ to determine $\omega_{0}\left(P^{\prime}\right)$

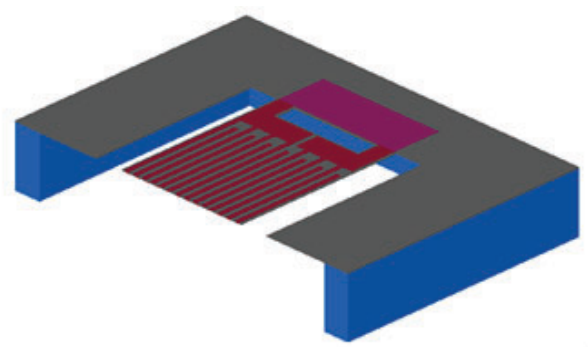

Figure 4: Half of the solid model to be used in Finite-element analysis. The model includes the resonator, support structure, undercut substrate, and portion of full thickness substrate.

TABLE I. KEY CHARACTERISTICS OF THE TWO RESONATORS

\begin{tabular}{|c|c|c|c|}
\hline Device Parameters & Symbol & Device 1 & Device 2 \\
\hline Frequency $(\mathrm{MHz})$ & $f_{0}$ & 456 & 567 \\
\hline Bottom Electrode Thickness $(\mathrm{nm})$ & $t_{P t}$ & 150 & 150 \\
\hline AlN Thickness $(\mu \mathrm{m})$ & $t_{A l N}$ & 1 & 1 \\
\hline Top Electrode Thickness $(\mathrm{nm})$ & $t_{A l}$ & 100 & 100 \\
\hline Pitch Width $(\mu \mathrm{m})$ & $W_{p}$ & 10 & 8 \\
\hline Resonator Length $(\mu \mathrm{m})$ & $L$ & 210 & 210 \\
\hline Number of Electrodes & $n$ & 7 & 7 \\
\hline
\end{tabular}

\section{6. $P C F=\left(\omega_{0}\left(P^{\prime}\right) / \omega_{0}^{0}-1\right) / P^{\prime}$}

The assumption of negligible acoustic loss to the substrate was shown to be valid for the AIN resonators in [6] resonating near 1 $\mathrm{GHz}$. If that is not the case then in step 2 the total heat applied, $P^{\prime}$, can be replaced with $P^{\prime \prime}=P^{\prime}(1-\alpha)$ where $\alpha$ is the fraction of acoustic power loss to the substrate.

\section{RESULTS}

We will demonstrate the methodology on two aluminumnitride (AIN) Laterally Vibrating Resonators (LVR) operating in their S0 mode similar to those described in [5] and shown in Fig. 3. The dimensions of the two resonators are shown in Table I.

The methodology described in the previous section was implemented in the CoventorWare ${ }^{\mathrm{TM}}$ commercial software suite [7]. Most of the steps in the power coefficient of frequency calculation as well as the nonlinearity modeling of Eq. 1 were automated within CoventorWare with the exception of a few steps as will become clear below.

The modeling domain shown in Fig. 4 includes not only the resonator, but also the support structure and undercut substrate. Modeling beyond the resonator is required because the etch that releases the resonator also undercuts the substrate and this undercut region has significant thermal resistance.

Next, we will demonstrate the details of the PCF method using the $567 \mathrm{MHz}$ resonator, and then present a comparison to measurement of the nonlinear response for both resonators.

\section{Modeling of PCF}

The model of Fig. 4 was meshed into 10555 parabolic piezoelectro-mechanical elements. 10215 of these elements were on the resonator and 340 on the support structure and substrate. For step 1 of the method described in the previous section, CoventorWare's FastPZE module was used to rapidly determine both the resonant frequency and its related displacement mode shape in the admittance response for the resonator at ambient temperature. Fig. 5(a) shows the S0 displacement mode shape, $|\boldsymbol{u}(\boldsymbol{r})|$. As expected, Fig. 5(b) shows the mode has a much larger displacement response near the center of the resonator compared to the portion near the anchors. 


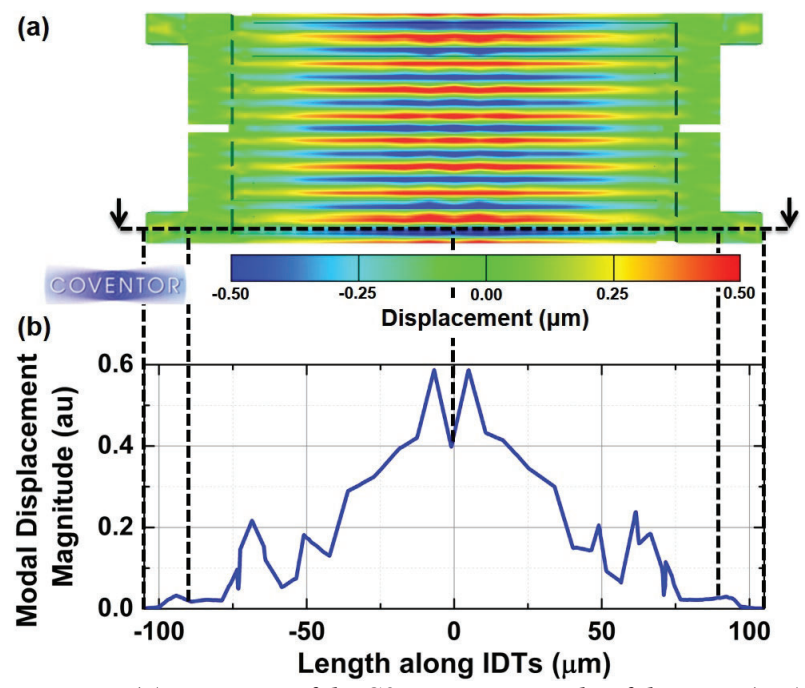

Figure 5: (a) Top view of the S0 operating mode of the LVR (top); (b) modal displacement amplitude along the indicated line.

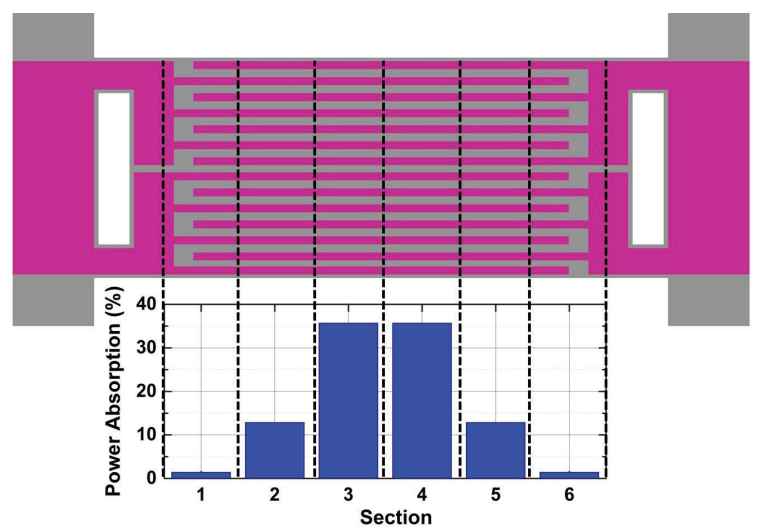

Figure 6: Approximate power absorption profile and boundaries of discretized zones for power input.

For step 2, a volume heat flux, $q(\boldsymbol{r})$, proportional to the square of the amplitude of the modal displacement field should automatically be applied to the model for the steady-state heat simulation. At the time of this writing, this automatic step was not available so a piecewise constant heat flux on 6 regions was applied that matches the square of the displacement field in form as shown in Fig. 6. This loss in detail did not significantly impact the PCF computation.

To compute the temperature distribution for step 3, the outer boundary of the substrate region is fixed at the ambient temperature of $293 \mathrm{~K}$ and the steady-state heat equation is solved with the heat flux of Fig. 6 scaled to give a total heat flux over the entire resonator of $P^{\prime}=1 \mathrm{~mW}$. The thermal conductivity for thin film aluminum nitride was used from [8]. The resulting temperature distribution shown in Fig. 7 shows a significant variation in temperature over the body of the resonator. This variation could not be modeled using a lumped method of a single temperature for the entire resonator.

For step 4, the thermo-acoustic coupling is obtained by using this temperature distribution to locally modify the stiffness tensor of aluminum nitride based on the temperature coefficients of stiffness from [9]. The resonance frequency is then recomputed with these distributed stiffness using the FastPZE module. The result for power levels of 1, 2, $4 \mathrm{~mW}$ is shown in Fig. 8 normalized to the resonance frequency. Based on $P=1 \mathrm{~mW}$, the PCF is $-360 \mathrm{ppm} / \mathrm{mW}$. The slight nonlinearity after $2 \mathrm{~mW}$ may be the result of thermal expansion or (a)

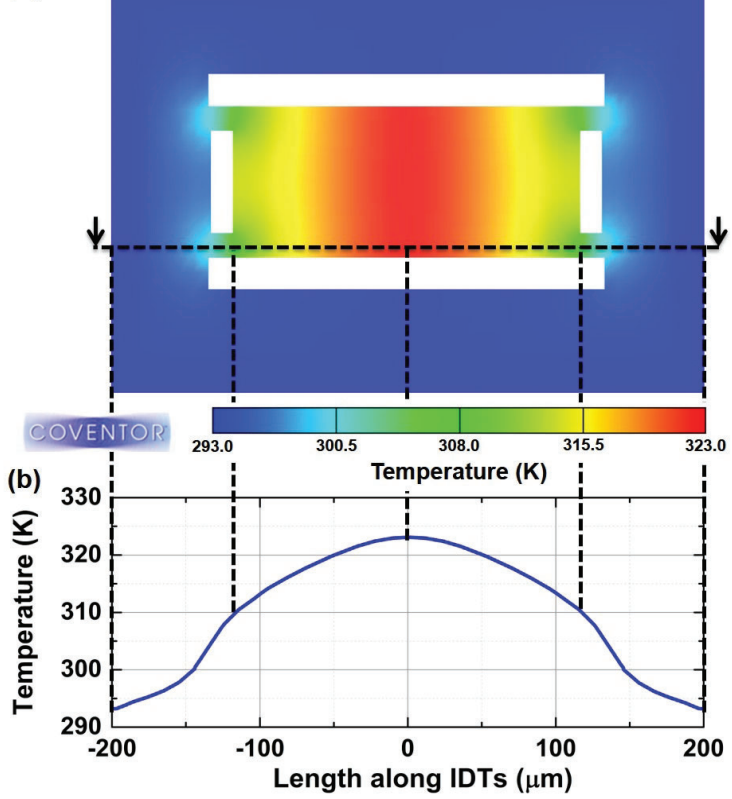

Figure 7: (a) Temperature field, $T(\boldsymbol{r})$, for $1 \mathrm{~mW}$ power absorbed; (b) temperature profile extracted along the indicated line.

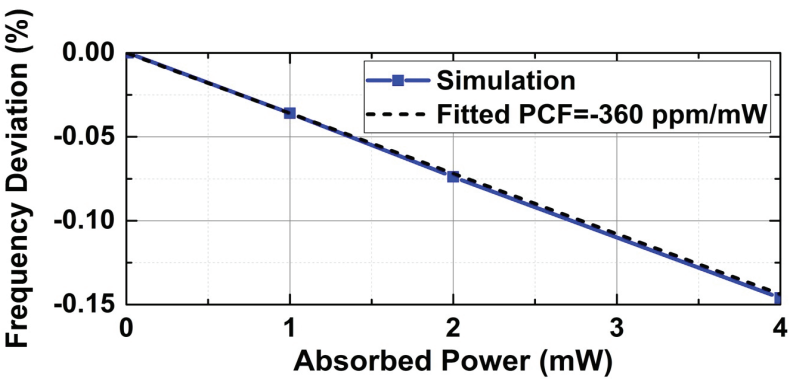

Figure.8: simulated frequency variation of the S0 mode as a function of the absorbed power for device 2. Frequency deviation is normalized to $f_{0}=566 \mathrm{MHz}$.

thermal stress that shifts the mechanical operating point of the structure.

The total computation time to perform this PCF calculation for $\mathrm{P}=1 \mathrm{~mW}$ was 15 minutes on a four-core computer with a clock speed of $2.70 \mathrm{GHz}$.

\section{Modeled and Measured Thermal Nonlinearity}

The PCF from the previous section was used to define $\omega_{0}(P)$ from Eq. 3. This definition of $\omega_{0}(P)$ was used in Eqs. 2 to determine $Z(\omega, P)$ from Fig. 2. Values for, $\omega_{0}^{0}, k_{t}^{2}, C_{0}$, and $Q$ for Eqs. 2 were taken from measured data rather than simulation to focus the following results on thermal nonlinearity modeling rather than the slight mismatch in simulated resonance frequency.

To determine $P(\omega)$ for a given $P_{a v s}$ and $Z_{0}$, Eq. 1 was solved for $P$ with Newton's method separately for each frequency point of interest. To capture hysteresis, the initial guess for $P$ is the solution for $P$ from the previous frequency point. Results for $P_{a v s}=1 \mathrm{dBm}$ are given in Fig. 9. As long as the first point in the upward or downward frequency sweep is outside the multi-valued region, the hysteresis will be properly captured.

With this $P(\omega)$, the admittance is computed as $\mathrm{Y}(\omega)=$ $Z(\omega, P(\omega))^{-1}$. The results for the two resonators are shown in Fig. 10 for $Z_{0}=50 \Omega$ and a series of increasing values of $P_{\text {avs }}$. For both resonators the lumped method severely under predicts the thermal 


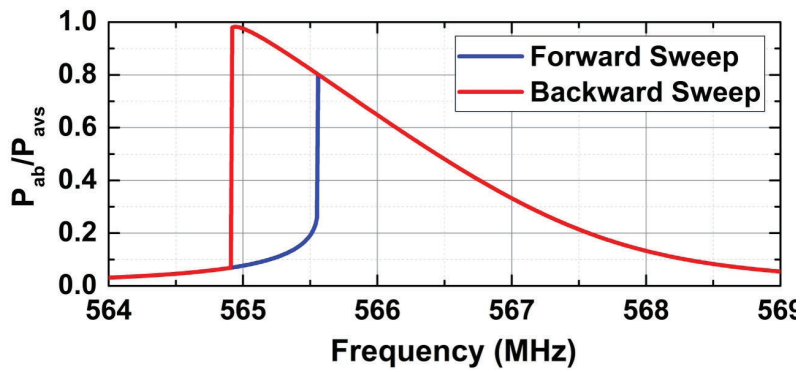

Figure 9: Power absorption as a function of frequency.
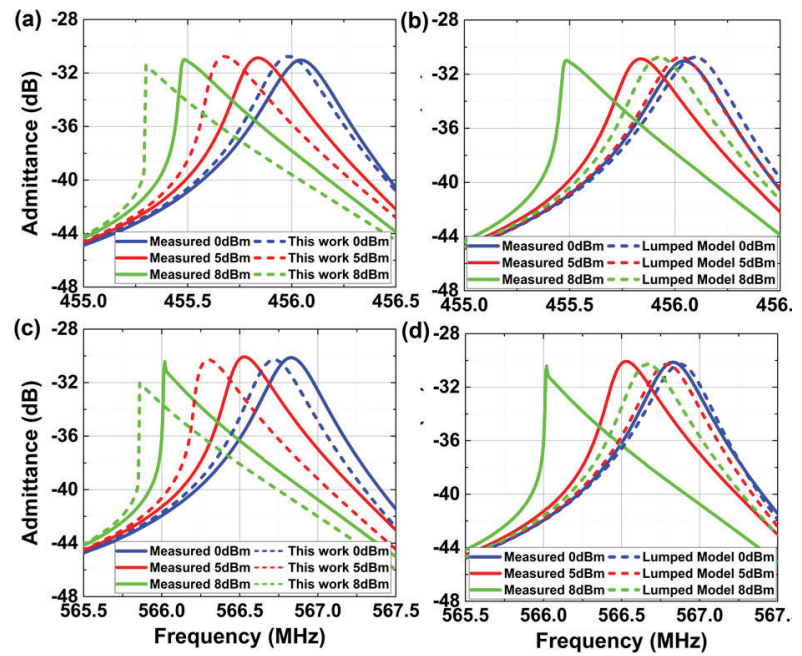

Figure 10: Measured admittance of device 1 compared with the (a) thermal-acoustically coupled model and (b) lumped model. Measured admittance of device 2 compared with (c) thermalacoustically coupled model and (e) lumped model. The measuement was done with an forward frequency sweep.

nonlinearity because it does not account for the higher thermal resistance for heat generated near the middle of the resonator. In contrast, the method of this paper shows a similar nonlinear response as the measurement, with some over prediction. The over prediction is expected since the portion of energy that is acoustically radiated into the substrate has not been subtracted from $P$ in the computation of PCF. Also, thin film thermal conductivity used from [8] is for deposition at $20{ }^{\circ} \mathrm{C}$, but the actual film was deposited at $300{ }^{\circ} \mathrm{C}$. Thin film sputter at higher temperature is expected to have better orientation and thus have conductivity closer to the higher bulk value than the value from [8].

\section{CONCLUSIONS}

In summary, this work presents a distributed and thermalacoustically coupled approach for accurate prediction of thermal nonlinearity in piezoelectric MEMS resonators. The methodology and detailed steps involved in the model have been outlined and explained. Two AlN resonators were used as the experimental testbed to validate the accuracy of the model. In comparison to the methods in prior work, the modeled results have shown closer match with the measurements. The modeling accuracy can be further improved by using measured thermal conductivity of the sputtered AlN thin-film and determining the proportion of energy acoustically radiated to the substrate. Despite being only showcased in AIN devices, the model herein can be readily extended to model thermal nonlinearity in any micro-scale resonators with temperature instability.

\section{REFERENCES}

[1] R. Lu and S. Gong, "Study of Thermal Nonlinearity in Lithium Niobate-Based MEMS Resonators," Solid-State Sensors, Actuators Microsystems Conf. (TRANSDUCERS), 2015 18th Int., pp. 8-11, 2015.

[2] J. Segovia-Fernandez and G. Piazza, "Thermal nonlinearities in contour mode AIN resonators," J. Microelectromechanical Syst., vol. 22, no. 4, pp. 976-985, 2013.

[3] R. Lu, A. Gao, and S. Gong, "Deciphering intermodulation in AlN laterally vibrating resonators," in Micro Electro Mechanical Systems(MEMS), IEEE 28th International Conference on, 2016, no. January, pp. 671-674.

[4] S. Wang, L. C. Popa, and D. Weinstein, "Piezoelectric nonlinearity in GaN Lamb mode resonators," in Solid-State Sensors, Actuators and Microsystems (TRANSDUCERS), 18th International Conference on, 2015, pp. 989-992.

[5] A. Gao and S. Gong, "Eradication of Asymmetric Spurious Mode in AlN MEMS Resonators using Mode Conversion of Lamb Waves," in IEEE 29th International Conference on Micro Electro Mechanical Systems (MEMS), 2016.

[6] J. Segovia-Fernandez, M. Cremonesi, C. Cassella, A. Frangi, and G. Piazza, "Anchor Losses in AIN Contour Mode Resonators," J. Microelectromechanical Syst., vol. 24, no. 2, pp. 265-275, Apr. 2015.

[7] Coventor, Inc, CoventorWare - MEMS simulation suite. Waltham, MA, http://www.coventor.com, 2015.

[8] S. R. Choi, D. Kim, S.-H. Choa, S.-H. Lee, and J.-K. Kim, "Thermal Conductivity of AIN and SiC Thin Films," Int. J. Thermophys., vol. 27, no. 3, pp. 896-905, Sep. 2006.

[9] J. H. Kuypers, C.-M. Lin, G. Vigevani, and A. P. Pisano, "Intrinsic Temperature Compensation of Aluminum Nitride Lamb Wave Resonators for Multiple-Frequency References," in Frequency Control Symposium, 2008, pp. 240-249. 